\title{
Investigating the Quality of Milk using Spectrometry Technique and Scattering Theory
}

\author{
Nur Ain Insyirah Muhamad Kamil \\ Advanced Devices and System (ADS) \\ Faculty of Engineering and Built Environment \\ Universiti Sains Islam Malaysia \\ Negeri Sembilan, Malaysia \\ Wan Zakiah Wan Ismail \\ Advanced Devices and System (ADS) \\ Faculty of Engineering and Built Environment \\ Universiti Sains Islam Malaysia \\ Negeri Sembilan, Malaysia \\ Juliza Jamaludin \\ Advanced Devices and System (ADS) \\ Faculty of Engineering and Built Environment \\ Universiti Sains Islam Malaysia \\ Negeri Sembilan, Malaysia
}

\author{
Zatunnur Syakirah Nor'aini \\ Advanced Devices and System (ADS) \\ Faculty of Engineering and Built Environment \\ Universiti Sains Islam Malaysia \\ Negeri Sembilan, Malaysia \\ Sharma Rao Balakrishnan \\ Advanced Devices and System (ADS) \\ Faculty of Engineering and Built Environment \\ Universiti Sains Islam Malaysia \\ Negeri Sembilan, Malaysia \\ Irneza Ismail \\ Advanced Devices and System (ADS) \\ Faculty of Engineering and Built Environment \\ Universiti Sains Islam Malaysia \\ Negeri Sembilan, Malaysia
}

\author{
Musab Sahrim \\ Advanced Devices and System (ADS) \\ Faculty of Engineering and Built Environment \\ Universiti Sains Islam Malaysia \\ Negeri Sembilan, Malaysia
}

\begin{abstract}
Milk is a dairy product that contains dissolved proteins, carbohydrates, fat, and many minerals. Milk enhances body growth and provides vital energy and fatty acids. Milk can turn bad after being kept at room temperature for several days. The endurance of milk could depend on its fat and protein composition. Our work aims to compare the quality of milk after being kept at room temperature for several days using spectroscopy methods. Modeling based on scattering theory is also provided to compare the light propagation in milk, water, and air. A VIS-NIR spectrometer was used to observe the light absorption, transmission, and reflectance whereas a modeling approach was applied to study the scattering, absorption, and extinction efficiencies. The milk samples consist of full cream milk kept at room temperature for 8 days, 11 days, 14 days, and 17 days. The results show that milk without fermentation has higher light absorbance and lower transmission compared to milk with fermentation, due to changes in milk composition after the fermentation process. Milk scatters more light compared to water and air due to its fat globule and protein ingredients. The output of this study can be used as a reference for studies involving bacteria or microorganisms in milk. It also can be used to compare the quality of milk with and without air exposure.
\end{abstract}

Corresponding author: Wan Zakiah Wan Ismail (drwanzakiah@usim.edu.my)
Keywords-light propagation; absorbance; transmittance; reflectance; scattering; milk; spectroscopy

\section{INTRODUCTION}

It is crucial to monitor the quality of milk in order to ensure we gain sufficient nutrients and minerals and prevent the occurrence of diseases. Cow milk consists of water (87\%), fat (4\%), proteins (3.4\%), lactose (4.8\%), and minerals (0.8\%) [1]. Fat composition is not similar in full cream milk and skimmed milk. A layer of cream forms on the milk's surface if it is exposed for several days. This cream consists of spheres of various sizes floating in milk surrounded by a fat globule membrane. The membrane is responsible for fat protection against enzymes and prevents any globule coalescing into butter grains [1]. The spectroscopy technique can be used to observe the optical properties of milk based on light absorbance, transmission and scattering. Mie scattering theory is used to compute the absorption coefficient $(\mu a)$, the scattering coefficient $(\mu s)$, and the phase function $p(\theta)$, where $\theta$ is the scattering angle [2]. Mie theory is used to calculate the spectral dependence for the extinction cross section of nanoparticle suspensions [3]. The pump source energy passes 
through the turbid media depending on optical properties such as the refractive index, scattering, anisotropic factor, and laser light absorption [4]. The optical properties of milk based on backscattering intensity can be used to study fat and protein concentrations [5]. The complex fluid of milk is made up of many components such as water, lipids, lactose and protein [6, 8]. Spectroscopy is widely used to measure the optical properties of samples based on light propagation and fluorescence. Color spectroscopy is used to obtain information about the atoms and molecules [8-9]. The absorbance spectroscopy is a technique used to measure the amount of absorbed light $[10,11]$ with the determination of solution concentration based on Beer's Law [12]. NIR spectrometer and VIS-NIR spectrometer with different wavelength ranges are used to determine the accuracy of the intensity spectrum in the spectroscopy analysis [13].

Many recent studies on light propagation in milk involve backscattering [5], external cavity-quantum cascade laser spectroscopy [6], and laser diffraction and centrifugation [7]. Authors in [8] introduced the simplified NIR spectroscopy in measuring the end of milk fermentation by transforming sugar to lactic acid. The key characteristic of the fermentation process is the $\mathrm{pH}$ end point value, in the range of 4.4-4.5 [8]. This technique is quite complicated and costly. To the best of our knowledge, no comparison has been conducted using milk after several days' exposure and water. The previous studies also do not provide a modeling approach on light scattering in milk. Our previous work [14] compared the optical properties of full cream and skimmed milk using different spectrometer types. We found that full cream milk has higher absorption due to its higher fat content. This research is continued in the current paper, which aims to study the light propagation in various milk samples for different exposure at room temperature durations based on spectroscopy techniques using Visible (VIS) and Near Infra-Red (NIR) spectrometers. The technique is simpler and cheaper than the ones used in previous studies as indicated in the experimental section. The output shows that the newly opened milk sample absorbs more light than the other samples. A modeling approach based on Mie theory was also provided to compare light scattering in milk, water, and air.

\section{THEORETICAL FRAMEWORK}

For the computation of Mie efficiencies, there are two input parameters which are the complex refractive index $m$ and the parameter size $x$ as shown in (1) and (2) [12].

$$
\begin{aligned}
& m=m^{\prime}+i m^{\prime \prime} \\
& x=k a=\frac{2 \pi}{\lambda} a
\end{aligned}
$$

where $\mathrm{m}^{\prime}$ is the real refractive index, $\mathrm{im}^{\prime \prime}$ is the imaginary refractive index, $k$ is wave number in the ambient medium, and $a$ is the sphere radius.

The key parameters of Mie theory are the computed amplitudes of the scattered field. The coefficients $a_{n}$ and $b_{n}$ are required to obtain the Mie efficiency using Spherical Bessel function $n(n=1,2, \ldots)$ of higher order and work well in the wider range of size parameters [15].
The efficiency of extinction $Q_{\text {ext }}$ and scattering $Q_{s c a}$ can be identified in forward-scattering theorem and in the integration of the power scatters in all directions. The absorption efficiency $Q_{a b s}$ can be identified with the equation of energy conservation [16]. Meanwhile, the backscattering efficiency $Q_{b}$ is applicable to monostatic radar [15]. Equations for absorption, scattering and backscattering efficiency are:

$$
\begin{gathered}
Q_{\text {ext }}=\frac{2}{x^{2}} \sum_{n=1}^{\infty}(2 n+1) \operatorname{Re}\left(a_{n}{ }^{2}+b_{n}{ }^{2}\right) \\
Q_{s c a}=\frac{2}{x^{2}} \sum_{n=1}^{\infty}(2 n+1)\left(\left|a_{n}\right|^{2}+\left|b_{n}\right|^{2}\right) \\
Q_{\text {ext }}=Q_{s c a}+Q_{a b s} \\
Q_{b}=\frac{1}{x^{2}}\left|\sum_{n-1}^{\infty}(2 n+1)(-1)^{n}\left(a_{n}-b_{n}\right)\right|^{2}
\end{gathered}
$$

where $x$ is the parameter size and $n$ is the spherical Bessel function order $n$.

The efficiency of radiation pressure can be proven by the Two-Stream Model and correlates with the asymmetry parameter [17].

$$
Q_{p r}=Q_{\text {ext }}+Q_{\text {sca }}(\cos \theta)
$$

where $\theta$ is the scattering angle.

Amplitude functions $S_{1}$ and $S_{2}$ indicate the scattering properties or the scattering of an electromagnetic wave from a spherical particle. The scattering function is required for the far field scatterer [16]:

$$
\begin{aligned}
& S_{1}(\cos \theta)=\sum_{n=1}^{\infty} \frac{2 n+1}{n(n+1)}\left(a_{n} \pi_{n}+b_{n} \tau_{n}\right) \\
& S_{2}(\cos \theta)=\sum_{n=1}^{\infty} \frac{2 n+1}{n(n+1)}\left(a_{n} \tau_{n}+b_{n} \pi_{n}\right)
\end{aligned}
$$

where

$$
\begin{aligned}
\pi_{n} & =\frac{2 n-1}{n-1} \cos \theta \cdot \pi_{n-1}-\frac{n}{n-1} \pi_{n-2} \\
\tau_{n} & =n \cos \theta \cdot \pi_{n}-(n+1) \pi_{n-1}
\end{aligned}
$$

\section{MethodOLODGY}

The research is conducted using experimental and theoretical methods. The light absorption and scattering analysis in milk are based on Mie scattering theory. The scattering, absorption, extinction, and backscattering efficiencies are analyzed in a homogeneous dielectric sphere and its angular scattering using MATLAB. The analysis is also repeated for water and air.

\section{A. Modeling Approach}

The modelling part is used to determine the characteristics of light in a disordered medium using MATLAB. The light propagation efficiency with the justification of Mie coefficient matrix is computed. The angular functions are also computed to produce the Mie angular efficiency. Figure 1 shows the flowchart of the constructed modeling approach. 


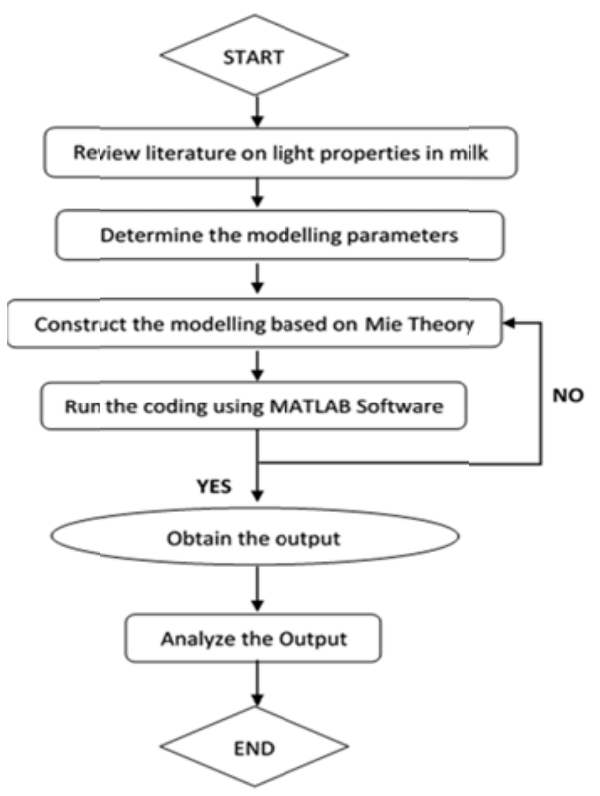

Fig. 1. The flowchart of the modeling approach.

\section{B. Experimental Approach}

The Ocean Optic Flame NIR spectrometer and VIS-NIR spectrometer were used to observe the characteristics of light propagation in milk. Every experiment was repeated 10 times to ensure the accuracy of the output. Five samples of milk with different days of exposure were used (the sample turns to yogurt after 14 days of air exposure). Figure 2 shows the flowchart of the experimental method.

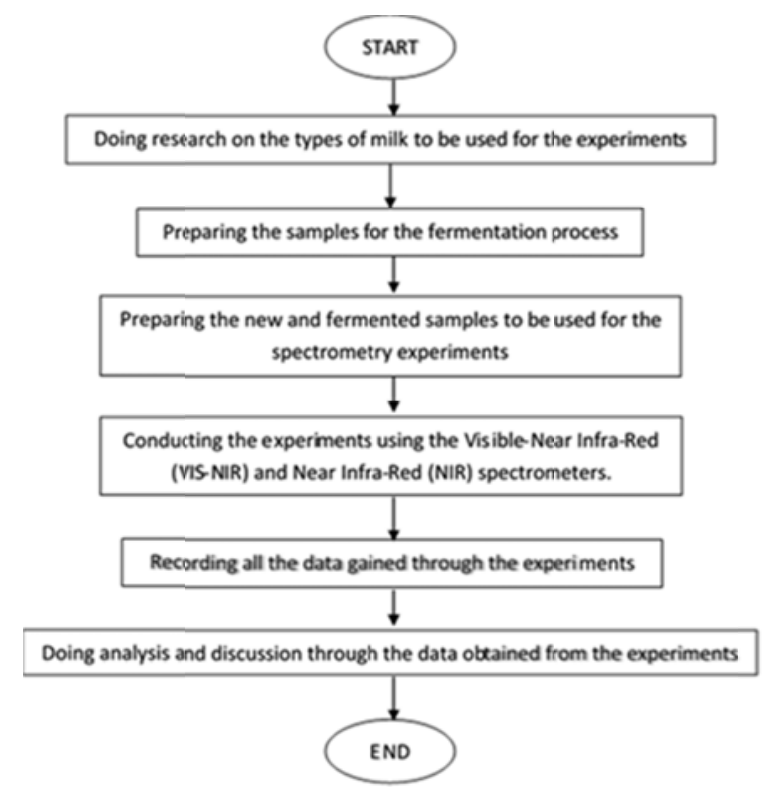

Fig. 2. The flowchart of the experiment.

The milk samples were kept at room temperature for 8,11 , 14 , and 17 days. The samples were diluted with a ratio of 1:100 of milk and water respectively. The prepared samples were placed in a cuvette of $10 \mathrm{~mm}$ and shaken lightly. The cuvette was placed into a cuvette holder and was illuminated by a halogen lamp (the light source). The light from the cuvette was collected by the VIS-NIR spectrometer (Figure 3). The spectrum graph was displayed on a computer screen using the OceanView software. Figure 4 shows the milk samples used in the experiments. Milk samples of 8 and 11 days were still cloudy but milk samples after 14 days became transparent with large milk particles observed.

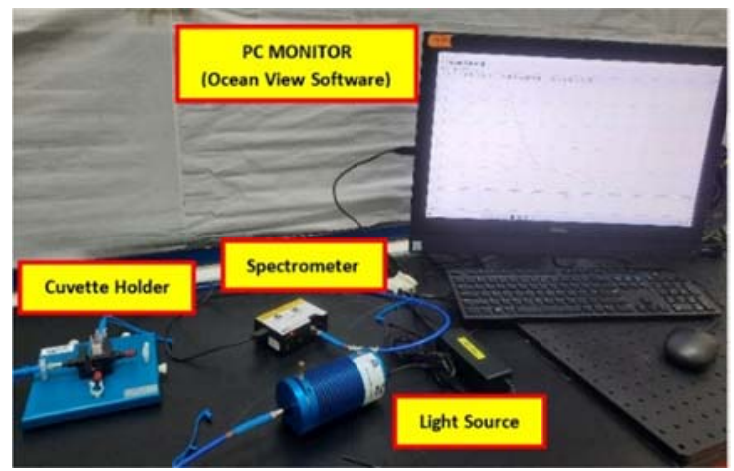

Fig. 3. The experimental set-up for the spectrometry technique.

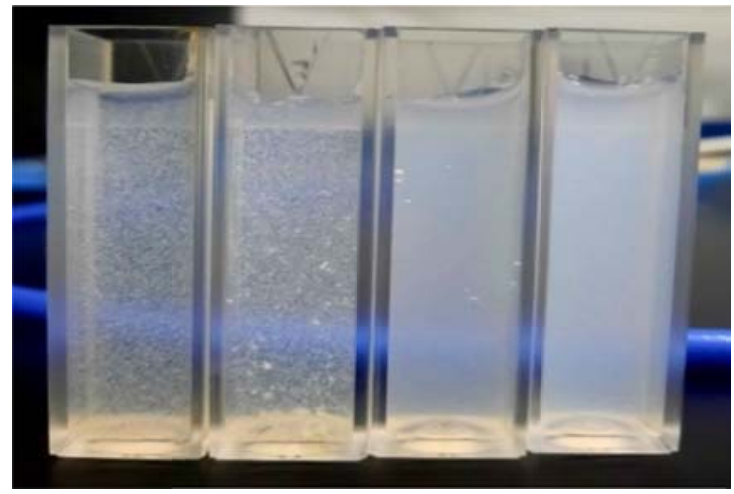

Fig. 4. Fermented full cream milk samples after dilution with water after $8,11,14$, and 17 days (from right to left) of exposure.

The set-up for reflectance spectroscopy is different from the absorbance and transmission spectroscopy setup. It can be used in liquid samples and solids. NIR spectrometer was used to observe the reflectance intensity spectrum of the samples. The samples were placed on the stage RTL-T. The reflectance spectrum of a sample in the cuvette was observed by a fiber probe. The probe transmits the light from the halogen light and then it was placed on the stage RTL-T. The reflectance spectrum can be observed by using a NIR spectrometer when the light propagates inside the medium.

\section{RESULTS AND DISCUSSION}

Previous works [5, 6] have analyzed fat and protein concentrations of milk through backscattering and spectroscopy. Authors in [7] studied the homogenization efficiency to control the development of the cream layer on fresh milk and authors in [8] used NIR spectroscopy to study yogurt fermentation automation. In this paper, we study the quality of milk which is kept at room temperature using VIS and NIR spectroscopy. We do a simple modeling to compare 
the light propagation in milk, water and air. The modeling analysis uses Mie theory to compute the efficiency of scattering, absorption, extinction, backscattering, asymmetry parameter, and radiation pressure whereas the experimental section shows the output in terms of absorbance, transmission, and reflectance. The output from the theoretical and experimental study are analyzed and discussed thoroughly in this section.

\section{A. Modeling based on Mie Scattering Theory}

The measurements of scattering, extinction and absorption efficiency based on Mie theory were conducted in MATLAB. The input parameters were the complex refractive index and the parameter size $x$ [18]. Modeling was done for milk, water, and air. Figures 5 and 6 summarize the modeling results.

(a)

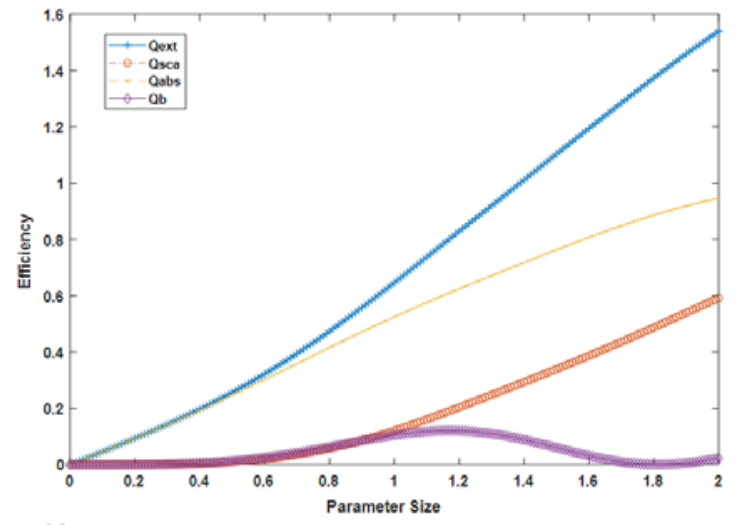

(b)

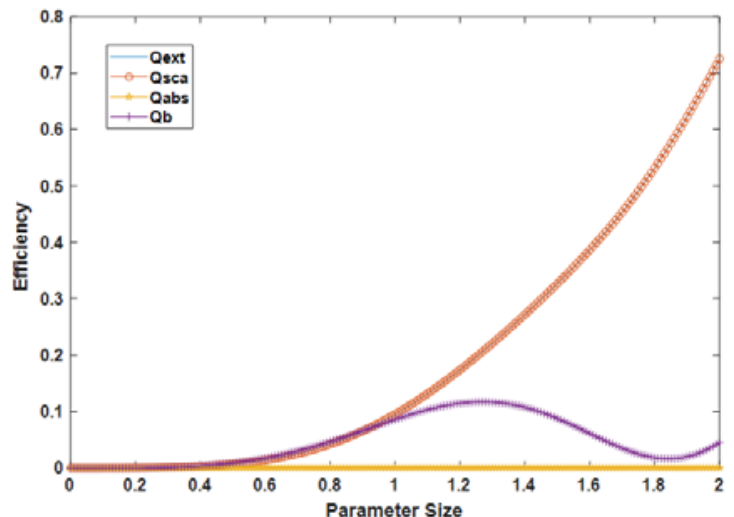

(c)

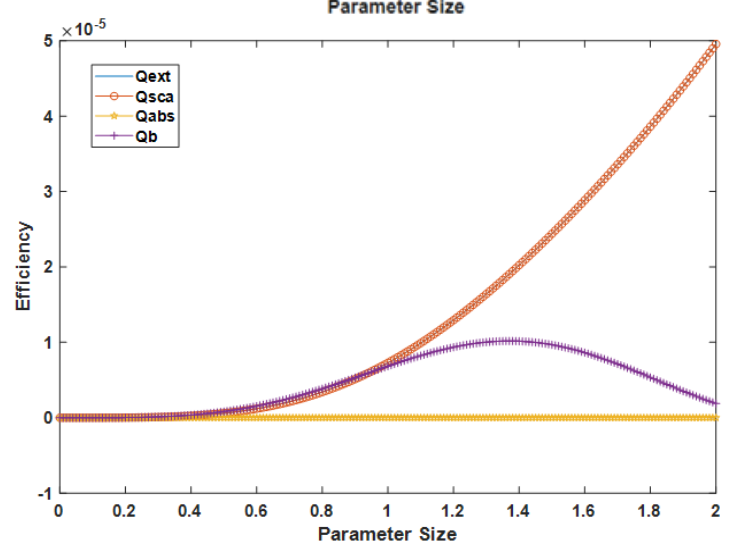

Fig. 5. Mie theory based efficiencies for (a) milk, (b)water, and (c) air.
Equations (3) to (7) were used in Figure 5. Figure 6 is plotted based on (8)-(9). The extinction, forward scattering, absorption, and backscattering efficiencies are represented by $\mathrm{Q}_{\mathrm{ext}}, \mathrm{Q}_{\mathrm{sca}}, \mathrm{Q}_{\mathrm{abs}}$ and $\mathrm{Q}_{\mathrm{b}}$ respectively. Figure 5 shows clearly that milk has better scattering efficiency than water and air. At parameter size 2, the scattering efficiency in milk (Figure 5(a)) reaches 0.6, while the scattering efficiency in water (Figure 5(b)) and air (Figure 5(c)) are 0.7 and $5 \times 10^{-7}$ respectively, prooving that the least light scattering occurs in the air, whereas milk and water consist of particles which can scatter the light. We presume that the light scattering and absorption are affected by the size and concentration of the particles, the incident light wavelength, and sample size [19]. Milk depicts the highest efficiency of light absorption due to its composition of fat globules and proteins. Figure 5 also shows that the forward scattering is more efficient compared to the backscattering for all samples due to the larger particles size of the samples. Figure 6 shows the scattering angle of milk, water and air respectively.

(a)

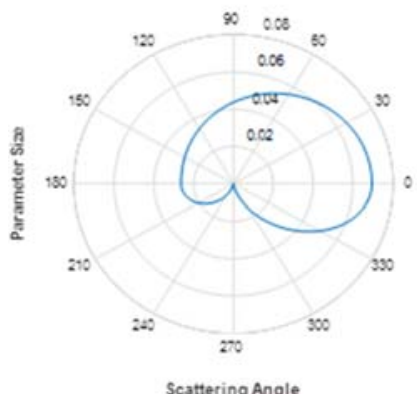

(b)

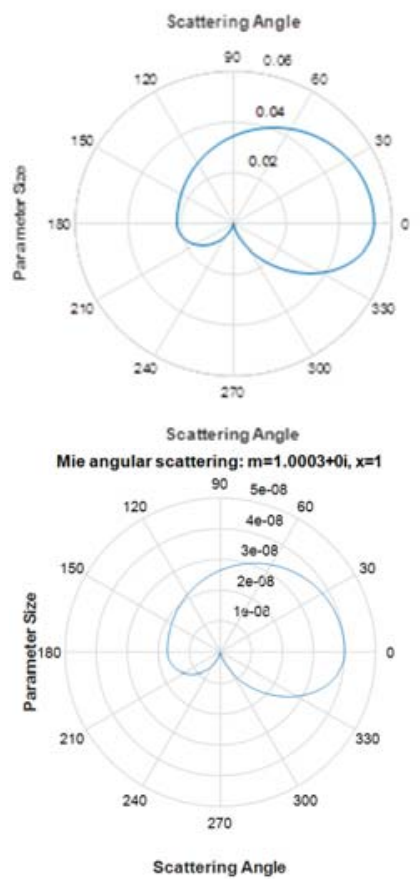

Fig. 6. Angular scattering for (a) milk, (b) water, and (c) air.

We observe that milk has larger value of angular scattering than water and air. It is clearly shown that milk has higher scattering effect. We attribute that to the milk contents which mostly consist of fat and proteins which can scatter light [20]. 


\section{B. Experimental Results}

The spectra of reflectance, transmission, and absorbance of the samples were monitored with the spectrometer. The samples of fresh full-cream milk were kept at room temperature for $8,11,14$, and 17 days. The experiment utilized an NIR Spectrometer and a VIS-NIR Spectrometer with wavelength ranges of $950 \mathrm{~nm}-1650 \mathrm{~nm}$ and $350 \mathrm{~nm}-1000 \mathrm{~nm}$ respectively. Figure 7 depicts the absorbance, transmission and reflectance spectra of the milk samples.

(a)

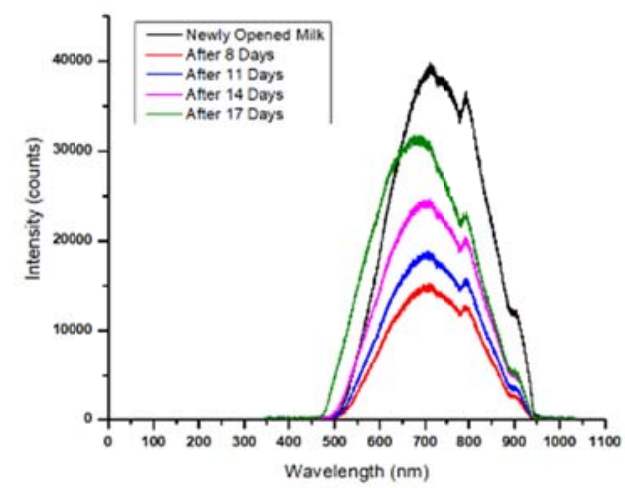

(b)

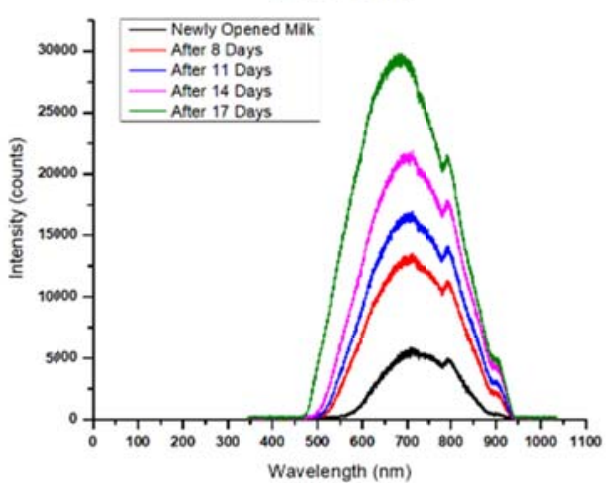

(c)

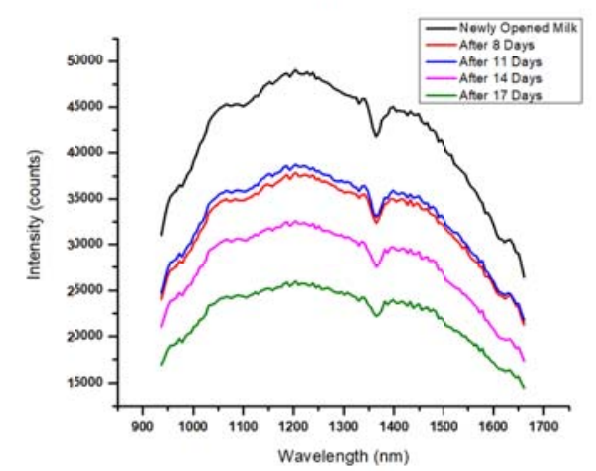

Fig. 7. (a) Absorbance, (b) transmission, and (c) reflectance of the milk samples for various days of fermentation.

Figure 7(a) shows the absorbance spectrum of the milk samples for different days of exposure. It is clearly shown that the absorbance spectra in freshly opened full-cream milk is higher than the milk that has been kept at room temperature for 17 days. The absorbance of milk reaches its peak at 700nm due to high attenuation coefficient which quickly absorbs the light around 700nm. When milk samples are kept at room temperature, they undergo physical changes where the particles aggregate and create lumpy which affect the light absorption [21] (see Figure 4). Hence, the quality from fresh to fermented milk is gradually decreasing as the protein concentration decreases due to milk coagulation [22].

(a)

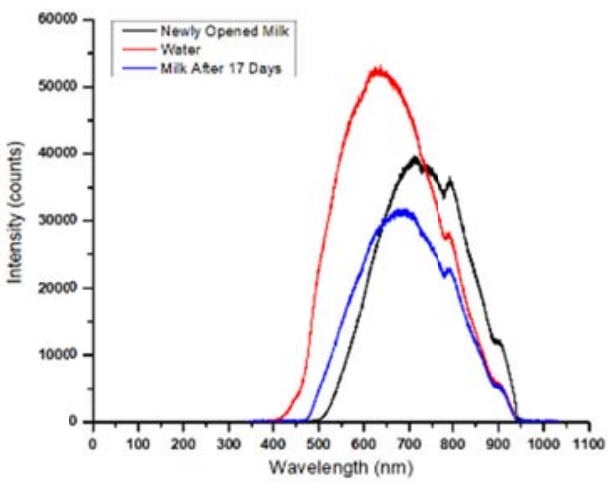

(b)

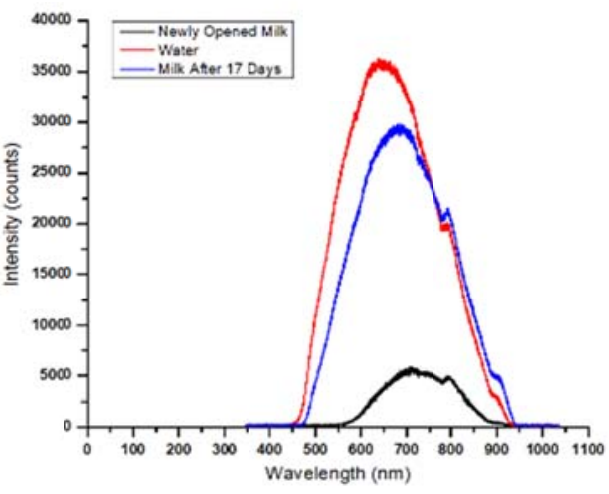

(c)

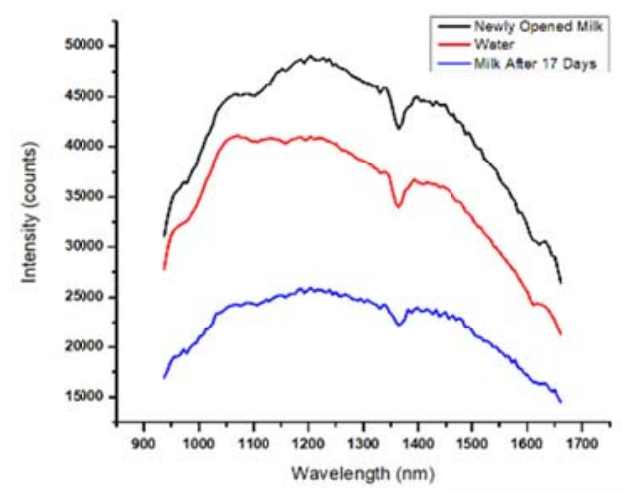

Fig. 8. (a) Absorbance, (b) transmission, and (c) reflectance of newly opened milk sample, water, and milk sample after 17 days.

Figure 7(b) shows the transmission spectra of freshly opened and fermented milks. The newly opened full-cream milk has lower light transmission than the fermented milk. The light transmission in fermented milk after 17 days is higher than in the rest of the samples as the particles in milk aggregate during the coagulation $[21,22]$. The protein thickens due to the fermentation process which produces lactic acid [23]. The fermented milk experiences decay and the $\mathrm{pH}$ level decreases as the lactic acid bacteria grow to produce the acid [24]. 
Figure 7(c) shows the reflectance spectra of the milk samples. The reflectance of newly opened milk is higher than the fermented milk's. The low reflectance values for fermented milk are recorded due to its high water absorption [25]. Hence, we suppose that the presence of fat globules and protein micelles in milk affect the light reflectance. The reflectance intensity decreases over the fermentation process due to the changes of protein and fat globules [26].

Figure 8 shows the spectra comparison of milk samples and water. Figure 8(a) shows that water absorbs most of the light at $600 \mathrm{~nm}$ whereas the absorption peak of milk is at 700nm. Milk and water depict similar transmission peaks at $\sim 650 \mathrm{~nm}$ (Figure 8(b)). Water sample shows higher transmission spectrum as it is more transparent than milk. Newly opened milk samples have higher reflectance than water due to their fat and protein composition. The size and shape of particles, the composition, and the concentration of the tested samples can affect the absorption, transmission, and reflectance of the samples respectively [26]. Newly opened milk samples consist of various particle compositions whereas the fermented milk samples have experienced physical state changes.

\section{CONCLUSION}

In conclusion, this research investigates the quality of milk for samples freshly opened and after being kept at room temperature for several days using spectroscopy and scattering theory. The optical properties of milk samples were investigated using VIS and NIR spectrometers. Newly opened milk samples have higher light absorbance and lower light transmission compared to the fermented milk, due to the aggregation of the fat and protein particles in milk during the fermentation process. Besides that, modeling based on scattering theory was done to compare the light propagation in milk, water, and air. The modeling shows that milk scatters more light compared to water and air due to the presence of fat globule, protein, and minerals. The outcome of the study shows that the quality of milk is reduced when it is kept at room temperature for several days. This is proved by both naked eye observation and spectroscopy. The outcome of this study can be useful in supporting future analysis studies on dairy products.

\section{ACKNOWLEDGEMENT}

We acknowledge the support of the Ministry of Education of Malaysia under the FRGS grant (FRGS/1/2018/STG02/ USIM/02/2) and Universiti Sains Islam Malaysia (USIM) for the funding and support.

\section{REFERENCES}

[1] G. H. Schmidt, L. D. V. Vleck, and M. F. Hutjens, Principles of Dairy Science, Subsequent edition. Englewood Cliffs, NJ, USA: Prentice Hall, 1988.

[2] A. Kienle, M. S. Patterson, L. Ott, and R. Steiner, "Determination of the scattering coefficient and the anisotropy factor from laser Doppler spectra of liquids including blood," Applied Optics, vol. 35, no. 19, pp. 3404-3412, Jul. 1996, https://doi.org/10.1364/AO.35.003404.

[3] A. A. Ramanenka, A. A. Lizunova, A. K. Mazharenko, M. F. Kerechanina, V. V. Ivanov, and S. V. Gaponenko, "Preparation and Optical Properties of Isopropanol Suspensions of Aluminum Nanoparticles," Journal of Applied Spectroscopy, vol. 87, no. 4, pp. 662-667, Sep. 2020, https://doi.org/10.1007/s10812-020-01051-w.
[4] M. A. Ansari, M. Erfanzadeh, and E. Mohajerani, "Mechanisms of Laser-Tissue Interaction: II. Tissue Thermal Properties," Journal of Lasers in Medical Sciences, vol. 4, no. 3, pp. 99-106, 2013.

[5] T. Katsumata, H. Aizawa, S. Komuro, S. Ito, and T. Matsumoto, "Quantitative analysis of fat and protein concentrations of milk based on fibre-optic evaluation of back scattering intensity," International Dairy Journal, vol. 109, Oct. 2020, Art. no. 104743, https://doi.org/10.1016/ j.idairyj.2020.104743.

[6] M. Montemurro, A. Schwaighofer, A. Schmidt, M. J. Culzoni, H. K. Mayer, and B. Lendl, "High-throughput quantitation of bovine milk proteins and discrimination of commercial milk types by external cavityquantum cascade laser spectroscopy and chemometrics," Analyst, vol. 144, no. 18, pp. 5571-5579, Sep. 2019, https://doi.org/10.1039/ C9AN00746F

[7] E. Ransmark, B. Svensson, I. Svedberg, A. Göransson, and T. Skoglund, "Measurement of homogenisation efficiency of milk by laser diffraction and centrifugation," International Dairy Journal, vol. 96, pp. 93-97, Sep. 2019, https://doi.org/10.1016/j.idairyj.2019.04.011.

[8] A. Aljaafreh and H. Steiner, "Evaluation of Using NIR Simplified Spectroscopy in Yogurt Fermentation Automation," presented at the 8th International Conference on Researches in Engineering, Technology and Sciences, Istanbul,Turkey, Aug. 2015.

[9] M. B. Alshammari, E. H. Anouar, and G. A. El-Hiti, "Spectroscopic Characterization, Hirshfeld Surface, DFT, and TD-DFT of tert-Butyl Phenethylcarbamate and 1,1-Dimethyl-3-Phenethylurea," Journal of Applied Spectroscopy, vol. 87, no. 4, pp. 736-744, Sep. 2020, https://doi.org/10.1007/s10812-020-01063-6.

[10] F. Alorifi, S. M. A. Ghaly, M. Y. Shalaby, M. A. Ali, and M. O. Khan, "Analysis and Detection of a Target Gas System Based on TDLAS \& LabVIEW," Engineering, Technology \& Applied Science Research, vol. 9, no. 3, pp. 4196-4199, Jun. 2019, https://doi.org/10.48084/etasr.2736.

[11] M. M. Nadareishvili, G. Mamniashvili, D. Jishiashvili, G. Abramishvili, C. Ramana, and J. Ramsden, "Investigation of the Visible LightSensitive ZnO Photocatalytic Thin Films," Engineering, Technology \& Applied Science Research, vol. 10, no. 2, pp. 5524-5527, Apr. 2020, https://doi.org/10.48084/etasr.3392.

[12] N. A. Bakar, Huize Cui, A. Abu-Siada, and Shengtao Li, "A review of spectroscopy technology applications in transformer condition monitoring," in 2016 International Conference on Condition Monitoring and Diagnosis (CMD), Xi'an, China, Sep. 2016, pp. 372-375, https://doi.org/10.1109/CMD.2016.7757895.

[13] R. A. Viscarra Rossel, D. J. J. Walvoort, A. B. McBratney, L. J. Janik, and J. O. Skjemstad, "Visible, near infrared, mid infrared or combined diffuse reflectance spectroscopy for simultaneous assessment of various soil properties," Geoderma, vol. 131, no. 1, pp. 59-75, Mar. 2006, https://doi.org/10.1016/j.geoderma.2005.03.007.

[14] N. A. I. Muhamad Kamil et al., "Investigating Light Propagation in Full and Skimmed Milk based on Spectroscopy and Monte Carlo Analysis," Journal of Engineering and Applied Science, vol. 67, no. 8, pp. 22652278, Dec. 2020

[15] C. Matzler, "MATLAB functions for Mie scattering and absorption," Institut für Angewandte Physik, Bern, Switzerland, Research Report 2002-08, Jun. 2002.

[16] K. L. van der Molen, "Experiments on scattering lasers: from Mie to random," Ph.D. dissertation, University of Twente, Enschede, Netherlands, 2007.

[17] W. E. Meador and W. R. Weaver, "Two-Stream Approximations to Radiative Transfer in Planetary Atmospheres: A Unified Description of Existing Methods and a New Improvement," Journal of the Atmospheric Sciences, vol. 37, pp. 630-643, Mar. 1980.

[18] Y. Ren, H. Qi, X. Yu, and L. Ruan, "A forward-angle-scattering method for the determination of optical constants and particle size distribution by collimated laser irradiation," Optics Communications, vol. 389, pp. 258-264, Apr. 2017, https://doi.org/10.1016/j.optcom.2016.12.060.

[19] D. J. Dahm, "Explaining Some Light Scattering Properties of Milk Using Representative Layer Theory," Journal of Near Infrared Spectroscopy, vol. 21, no. 5, pp. 323-339, Oct. 2013. 
[20] L. C. Andrews, Field Guide to Atmospheric Optics, Second Edition, 2nd ed. Washington, DC, USA: SPIE--The International Society for Optical Engineering, 2019.

[21] C. Hahn, M. Sramek, S. Nöbel, and J. Hinrichs, "Post-processing of concentrated fermented milk: influence of temperature and holding time on the formation of particle clusters," Dairy Science \& Technology, vol. 92, no. 1, pp. 91-107, Jan. 2012, https://doi.org/10.1007/s13594-0110046-1.

[22] S. L. Thomsen, S. L. Jacques, and S. T. Flock, "Microscopic correlates of macroscopic optical property changes during thermal coagulation of myocardium,” in Laser-Tissue Interaction, Jun. 1990, vol. 1202, pp. 2 11, https://doi.org/10.1117/12.17605.

[23] M. Lu et al., "Milk Spoilage: Methods and Practices of Detecting Milk Quality," Food and Nutrition Sciences, vol. 4, no. 7, pp. 113-123, Jul. 2013, https://doi.org/10.4236/fns.2013.47A014.

[24] R. Fernandes, Ed., Microbiology Handbook: Dairy Products, 3rd ed. Cambridge, UK: Royal Society of Chemistry, 2009.

[25] B. Aernouts, E. Polshin, J. Lammertyn, and W. Saeys, "Visible and nearinfrared spectroscopic analysis of raw milk for cow health monitoring: Reflectance or transmittance?," Journal of Dairy Science, vol. 94, no. 11, pp. 5315-5329, Nov. 2011, https://doi.org/10.3168/jds.2011-4354.

[26] J. A. Raty and K.-E. Peiponen, "Reflectance Study of Milk in the UVVisible Range," Applied Spectroscopy, vol. 53, no. 9, pp. 1123-1127, Sep. 1999. 